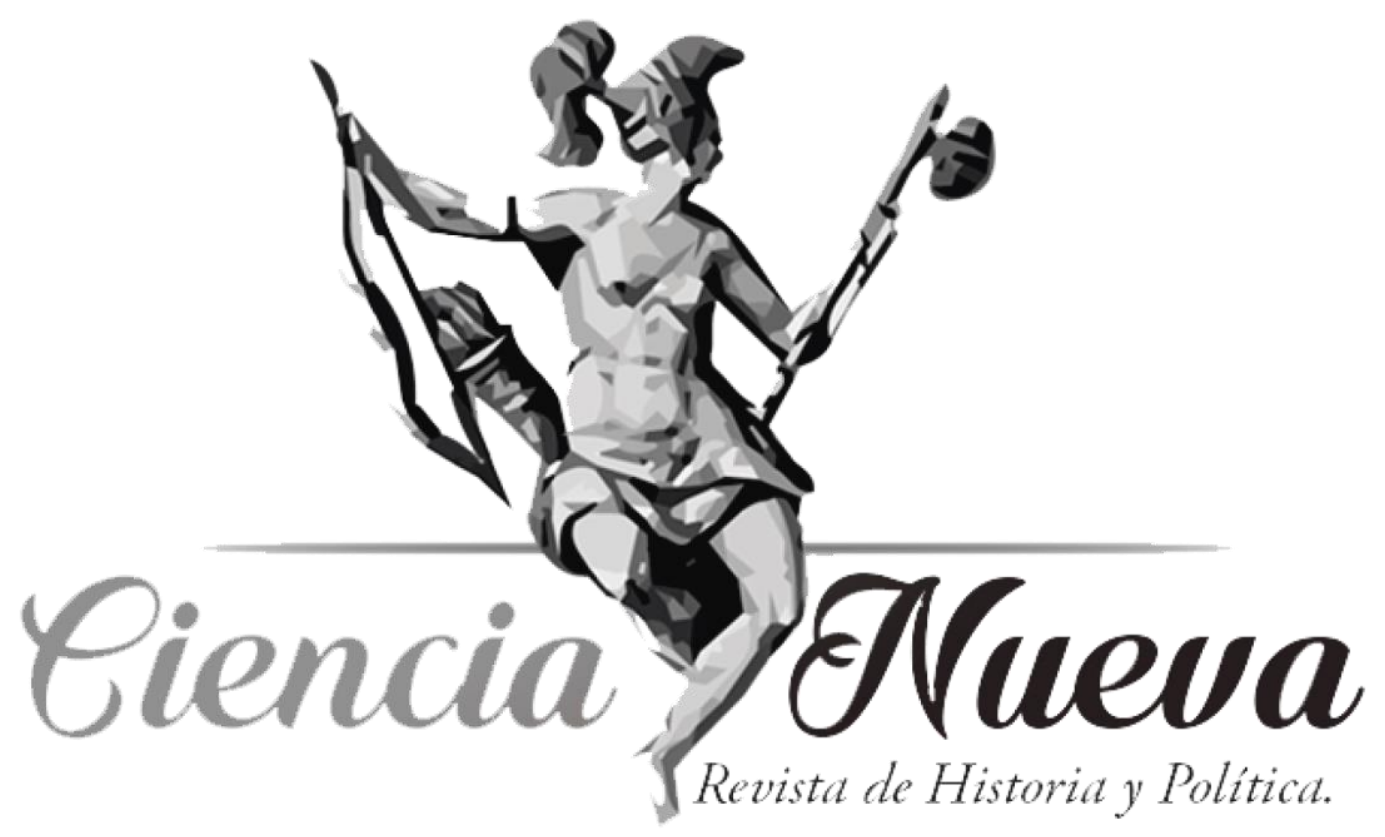

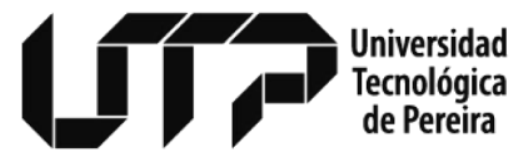

Maestría en Historia

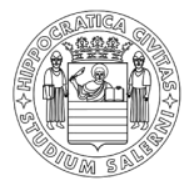

UNIVERSITÀ DEGLI STUDI DI SALERNO

Maestría en Ciencia Política

RESEÑAS

JHON JAIME CORREA, NATALIA AGUDELO Y CHRISTIAN NIÑO. FACULTAD DE CIENCIAS DE LA EDUCACIÓN DE LA UTP (1967-2017). 50 AÑOS EN LA CONSTRUCCIÓN DE UN PROYECTO EDUCATIVO PARA UNA NUEVA REGIÓN. PEREIRA: EDITORIAL UTP, 2018 JHON JAIME CORREA, NATALIA AGUDELO Y CHRISTIAN NIÑO. FACULTY OF EDUCATION SCIENCES OF THE UTP (1967-2017). 50 YEARS BUILDING AN EDUCATIONAL PROJECT FOR A NEW REGION. PEREIRA: EDITORIAL UTP, 2018 DOI: https://doi.org/10.22517/25392662.21821

Wilton Holguín Rotavista pp. $188-190$

Vol. 3 Núm. 2 | Julio-Diciembre de 2019

Pereira, Colombia 


\title{
Jhon Jaime Correa, Natalia Agudelo y Christian Niño. Facultad de CIENCIAS DE LA EDUCACIÓN DE LA UTP (1967-2017). 50 AÑOS EN LA CONSTRUCCIÓN DE UN PROYECTO EDUCATIVO PARA UNA NUEVA REGIÓN. PEREIRA: EDITORIAL UTP, 2018*
}

\author{
JHON JAIME CORREA, NATAlia AgUdelo Y CHRISTIAN NiÑO. FACULTY OF EDUCATION \\ SCIENCES OF THE UTP (1967-2017). 50 YEARS BUILDING AN EDUCATIONAL PROJECT FOR A NEW \\ REGION. PEREIRA: EDITORIAL UTP, 2018
}

Wilton Holguín Rotavista**

wilton.211513210@ucaldas.edu.co

ORCID: http://orcid.org/0000-0001-7868-480X

\begin{aligned} & \hline Recibido: 25 de marzo de 2019. \\ & Revisado: 28 de junio de 2019. \\ & Aceptado: 18 de septiembre de 2019. \\ & Publicado: 30 de diciembre de 2019. \\ & \hline\end{aligned}

$\mathrm{E}$ 1 libro de Jhon Jaime Correa, Natalia Agudelo y Christian Javier Niño se ocupa de la Facultad de Educación de la Universidad Tecnológica de Pereira y de la construcción de su proyecto educativo durante 50 años (1967-2017). La obra, que hace parte de la colección de la Maestría en Historia, es el resultado del proyecto de investigación financiado por la Vicerrectoría de Investigaciones, Innovación y Extensión de la Universidad Tecnológica de Pereira.

Para lograr el análisis histórico-crítico y contextualizado que se proponen, los autores dividen el libro en cinco capítulos.

En el primer capítulo, “Aproximación a un contexto histórico cambiante, dinámico y conflictivo", se hace un recuento de los procesos de modernización del sistema educativo colombiano, específicamente de la educación superior, desde los inicios de la posguerra (1945) hasta los años del pacto bipartidista del Frente Nacional (1958-1974) ${ }^{1}$. En este apartado, los autores argumentan que la creación de la Universidad Tecnológica de Pereira responde a la necesidad del país de impulsar el desarrollo y la industrialización en las regiones, consigna compartida por un sector de la élite pereirana que promovió el proyecto hasta materializarlo. "Las élites y las comunidades de interpretación"”2 son temas de interés de Jhon Jaime Correa y del grupo de investigación Políticas, Sociabilidades y Representaciones Histórico-Educativas (PSORHE), al que pertenecen los tres autores.

El segundo capítulo, "Los primeros años de la FCE: entre motivaciones e incertidumbres", aborda la creación de la FCE, en una universidad que en sus primeros años

\footnotetext{
* Este documento respeta las directrices y normas dispuestas en la Declaración de Ética de Publicación de Ciencia Nueva, Revista de Historia y Política. Esta declaración puede consultarse en la página web de la revista: http://revistas.utp.edu.co/index.php/historia

${ }^{* *}$ Estudiante de Licenciatura en Ciencias Sociales de la Universidad de Caldas.

${ }^{1}$ Jhon Jaime Correa Ramírez, Natalia Agudelo Castañeda y Christian Javier Niño Posada, Facultad de Ciencias de la Educación de la UTP (1967-2017). 50 años en la construcción de un proyecto educativo para una nueva región (Pereira: Editorial UTP, 2018), 20.

${ }^{2}$ Correa, Agudelo y Niño, La Facultad ...46
} 
se enfocó en la educación tecnológica e ingenieril. De otro lado, ante la falta de docentes a nivel básico y secundario en el recién creado departamento de Risaralda se evidenció la necesidad de abrir nuevos programas para atender a la demanda de profesionalización docente $^{3}$. Es válido mencionar que durante este periodo la organización del movimiento universitario por primera vez planteó una dimensión amplia de problemáticas acerca del papel del sistema educativo en nuestro país y en contra de la estructura jerárquica de la Universidad $^{4}$.

En el periodo que trata el tercer capítulo, "Transformaciones estructurales: La FCE entre las décadas del setenta y ochenta", toma importancia la discusión ideológica que se dio en los claustros universitarios del país entre los partidos tradicionales y las nuevas organizaciones políticas de izquierda como un reflejo de las tensiones ideológicas propias de la Guerra Fría ${ }^{5}$. En este ambiente de movilización y protesta se registraron fuertes disturbios en la UTP, que se agudizaron tras la decisión de las directivas de expulsar a líderes estudiantiles y profesores; estas problemáticas originaron el cierre de la universidad por cuatro meses 6 .

"Década de los noventa: Una época en crisis" es el nombre del capítulo que da cuenta de un periodo donde se manifestó, aún más, la desfinanciación, la expansión de la cobertura educativa, el aumento de los programas de operación comercial y la disminución de la planta docente $^{7}$. En este apartado los autores demuestran cómo la Constitución Política de 1991, la Ley General de Educación de 1994, la apertura económica y la intensificación de las políticas de calidad originaron cambios estructurales en las facultades de educación del país. Los autores ponen en evidencia los anhelos, las frustraciones y las inercias de instituciones como estas dependencias académicas que fundamentan sus acciones en ser formadoras de formadores.

El último capítulo del libro, "Una mirada crítica a la historia reciente de la facultad", es una radiografía de la situación actual de la universidad pública colombiana. En esta parte se logra entender cómo una facultad que surgió dentro de un proyecto de universidad ingenieril se convirtió en la facultad con mayor oferta y demanda de posgrados; en este capítulo final se plantean varios interrogantes que las universidades públicas deberán resolver frente a proyectos recién aprobados o que están en proceso de aprobación y que responden a la necesidad de masificar la educación pública.

Como se ha mencionado, el libro sobre la Facultad de Ciencias de la Educación tiene un interés particular en analizar la facultad desde el contexto; sin embargo, este enfoque que permite entender que la historia de la facultad no se ha desarrollado en un vacío, alejado del ámbito cultural, político, económico y jurídico del país y de la región, en ocasiones, da la impresión de que los autores enfatizan de más en el contexto. Pero esto último es justificable en tanto que su objeto de estudio se modifica en la medida que logra entablar una conversación entre las políticas educativas del país, las necesidades de comunidades regionales y las estrategias, los métodos y los paradigmas de cada una de las áreas de conocimiento que componen los programas formativos de la Universidad.

\footnotetext{
${ }^{3}$ Correa, Agudelo y Niño, La Facultad..., 20.

${ }^{4}$ Correa, Agudelo y Niño, La Facultad..., 90.

${ }^{5}$ Correa, Agudelo y Niño, La Facultad..., 119.

${ }^{6}$ Correa, Agudelo y Niño, La Facultad..., 121.

${ }^{7}$ Correa, Agudelo y Niño, La Facultad..., 194.
} 
De acuerdo con lo anterior, se hace necesario reconocer que el trabajo de Correa, Agudelo y Niño es ambicioso toda vez que pretende analizar un objeto de estudio que se adapta a las demandas de diferentes actores y donde, en un periodo muy extenso, se quiso involucrar en el análisis a cada uno de estos. Pero esta ambición, fundamentada quizás en las riqueza de las fuentes, obligó a darle menos importancia a algunos procesos y actores durante el desarrollo de ciertos capítulos con el fin de profundizar, según los autores, en las característica más importantes de cada periodo; es así como, por ejemplo, en el capítulo dos, "Los primeros años de la FCE: entre motivaciones e incertidumbres (1960-1973)", le dan prioridad a los procesos internos de la facultad relevando el contexto global que se venía desarrollando y al que vuelven, plenamente, en el último capítulo "Una mirada crítica a la historia reciente de la facultad".

Por último, una de las características del libro que lo hace novedoso en la región ${ }^{8}$ es el uso y la variedad de fuentes que utilizan los autores para alcanzar el objetivo propuesto; porque, si bien el objeto de estudio cuenta con un archivo institucional que respalda 50 años de labores, los autores incorporaron a su discurso fuentes externas que enriquecen el análisis, y en el mismo involucran actores que defendieron posturas diferentes durante la construcción del proyecto educativo de la UTP, reconociendo así que las instituciones no son solamente formas o estructuras jurídicas, sino que además interactúan en el contexto social con base en sus premisas jurídicas, pero atendiendo a las influencias de las problemáticas externas ${ }^{9}$. Algunas de las fuentes utilizadas fueron el archivo del sindicato (ASPU), normativas nacionales, la prensa y las fuentes orales, estas últimas esenciales en la construcción de la historia reciente de la facultad, la cual permitió concluir que una facultad formadora de formadores en la actualidad deberá reevaluar su proyecto educativo y retomar con más vigor el debate permanente sobre el perfil profesional que pretende que construyan los estudiantes $^{10}$, incitando a un continuo ejercicio reflexivo sobre la educación.

\footnotetext{
${ }^{8}$ En la región del Viejo Caldas el único trabajo que se había publicado con objetivos similares fue el de Albeiro Valencia Llano sobre la Universidad de Caldas. Sin embargo, aunque ambas investigaciones tienen el objetivo de historiar instituciones dedicadas a la formación, los trabajos distan mucho en enfoque, fuentes y presupuestos teóricos.

${ }^{9}$ Correa, Agudelo y Niño, La Facultad...,18.

${ }^{10}$ Correa, Agudelo y Niño, La Facultad..., 229.
} 